\title{
Low Cost SIW Chebyshev Bandpass Filter with New Input/Output Connection
}

\author{
Augustine O. Nwajana, Kenneth S. K. Yeo, Amadu Dainkeh \\ Department of Electrical and Electronic Engineering \\ University of East London \\ London, UK \\ a.nwajana@ieee.org
}

\begin{abstract}
This paper presents a substrate integrated waveguide (SIW) Chebyshev bandpass filter using the low cost, commercially available printed circuit board (PCB) technology. The detailed design procedure beginning from the normalized Chebyshev lowpass filter, to the final optimized SIW bandpass filter is presented. The test filter having a $4 \%$ fractional bandwidth centered at $1.684 \mathrm{GHz}$ was fabricated on a $1.27 \mathrm{~mm}$ thick, Rogers RT/Duroid 6010LM substrate with a 10.8 dielectric constant. The design has also been experimentally validated and results presented. The simulation and measurement responses of the filter show good agreement with a low insertion loss of $1.3 \mathrm{~dB}$. The simulated and the measured return losses of about $15 \mathrm{~dB}$ and $16 \mathrm{~dB}$ respectively, were achieved across the filter passband.
\end{abstract}

Keywords-bandpass filter; coupling; substrate integrated waveguide (SIW); cavity resonators; coplaner waveguide (CPW)

\section{INTRODUCTION}

Substrate integrated waveguide (SIW) is a type of transmission line that has evolved in the past decade. This new transmission line has come to bridge the gap between traditional air-filled waveguides and planar transmission lines such as microstrip. Traditional air-filled rectangular waveguide can be utilized in the design of high-performance microwave filters but requires complex transition to integrate planar circuits, not to mention its' bulky size. A better solution is to integrate the conventional rectangular waveguide into the microstrip substrate, giving rise to the SIW [1]. Though the above integration of the rectangular waveguide into the microstrip will surely reduce the Q-factor of the waveguide due to dielectric filling and volume reduction, the whole circuit (including planar circuit, transition, and waveguide) can be manufactured using standard printed circuit board (PCB) as indicated in [2], or by using any other multi-layer processing methods including the low temperature co-fired ceramic (LTCC) technique [3]-[7], and the liquid crystal polymer (LCP) technique [8].

Bandpass filters are very useful and popular when it comes to achieving complex and multi-port devices and systems. A bandpass filter (BPF) is a device that passes frequencies within a single band while rejecting all other frequencies outside the band [9]. The design parameters of filters such as selectivity, cost, miniaturization, sensitivity to environmental effects, power handling capacity, in-band and out-of-band performance metrics, are critical specifications when it comes to the development of radio frequency (RF) and microwave front ends. Designers are often required to make compromise between several conflicting requirements as it is rather difficult or even physically and/or electrically impossible to simultaneously achieve some design criteria or specifications. For instance, achieving higher channel selectivity usually requires the use of more resonators, which means higher insertion loss along the transmission path because insertion loss is approximately proportional to the number of resonators used in the construction of a filter [10].

\section{SIW CAVITY}

Fig. 1 represents a typical SIW cavity (or resonator) constructed entirely on a dielectric substrate with a thickness of h. The length and width of the cavity are indicated as $l$ and $w$ respectively. The value of $\mathrm{h}$ is usually smaller than that of $w$ (that is: $h<w$ ). The top and bottom walls of the cavity are made of metallic plates on both sides of the substrate. The side walls are formed by metallic posts (or vias). The vias have a diameter indicated as $d$, and the separation between adjacent vias (also known as the pitch length) is indicated as $p$. To minimize the radiation loss in a cavity, $d$ and $p$ values must be carefully chosen. In [11], it was explained that the radiation loss is negligible with a ratio $\mathrm{d} / \mathrm{p}$ of 0.5 for an electrically small via. In general, the radiation loss tends to decrease as the via gets smaller for a constant ratio $d / p$, which is conditioned by the fabrication process [12].

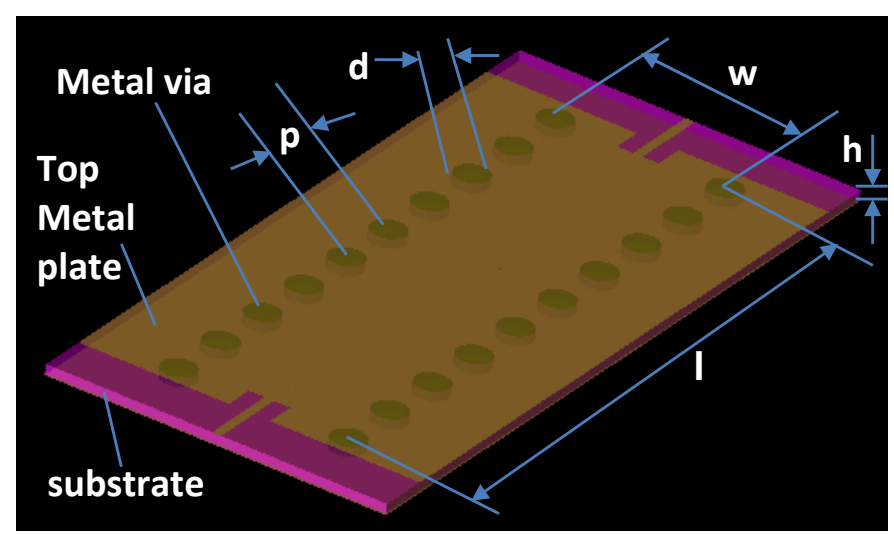

Fig. 1. Substrate integrated waveguide cavity.

The size of an SIW cavity, for the dominant $\mathrm{TE}_{101}$ mode, is determined by the corresponding resonance frequency, $f_{10}$ 
from [13] using (1), where $w_{\text {eff }}$ and $l_{\text {eff }}$ are the equivalent width and length of the SIW cavity, $\mu_{r}$ is the relative permeability (or dielectric constant) of the substrate, and $c_{0}$ is the speed of light in free space.

$$
\begin{gathered}
f_{101}=\frac{c_{0}}{2 \pi \sqrt{\mu_{r} \varepsilon_{r}}} \sqrt{\left(\frac{\pi}{w_{\text {eff }}}\right)^{2}+\left(\frac{\pi}{l_{\text {eff }}}\right)^{2}} \\
w_{\text {eff }}=w-1.08 \frac{d^{2}}{p}+0.1 \frac{d^{2}}{w}, \\
l_{\text {eff }}=l-1.08 \frac{d^{2}}{p}+0.1 \frac{d^{2}}{l}
\end{gathered}
$$

\section{BANDPASS FILTER CIRCUIT MODEL}

The filter presented in this paper was designed with the following specifications: center frequency $\mathrm{f}_{0}, 1.684 \mathrm{GHz}$; fractional bandwidth FBW, 4\%; and passband return loss RL, $20 \mathrm{~dB}$. The circuit model of the BPF was established from the normalized lowpass filter shown in Fig. 2. The normalized Chebyshev lowpass filter parameters $g$, at $0.04321 \mathrm{~dB}$ passband ripple, were determined from [12] and presented in Table I. Admittance inverters (J-inverters) were utilized in transforming the normalized lowpass filter to a shunt-only network as shown in Fig. 3. Each shunt capacitors in Fig. 3 is made to be equal to $g_{I}[14]$.

TABLE I. CHEBYSHEV LOWPASS FILTER PARAMETERS

\begin{tabular}{|l|r|r|r|r|}
\hline $\mathbf{g}_{\mathbf{0}}$ & $\mathbf{g}_{\mathbf{1}}$ & $\mathbf{g}_{\mathbf{2}}$ & $\mathbf{g}_{\mathbf{3}}$ & $\mathbf{g}_{\mathbf{4}}$ \\
\hline 1.0 & 0.8516 & 1.1032 & 0.8516 & 1.0 \\
\hline
\end{tabular}

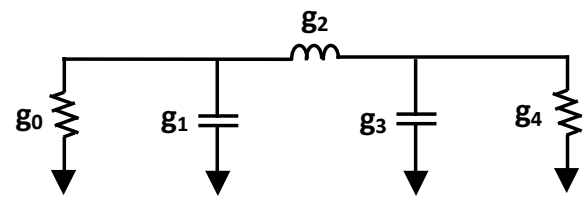

Fig. 2. Standard Normalized $3^{\text {rd }}$ order lowpass filter.

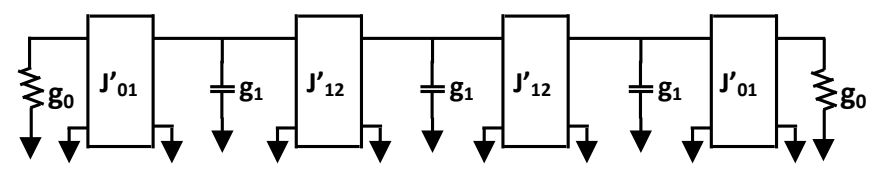

Fig. 3. Normalized lowpass filter with J-inverters and shunt-only components.

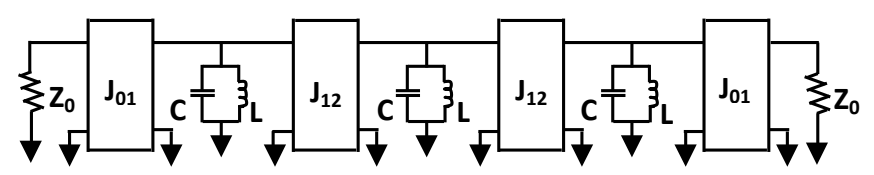

Fig. 4. Chebyshev bandpass filter circuit model with identical LC resonators and J-inverters.
The J-inverter values were calculated by using (2), where $n$ is the order of the filter and $m=1,2, \ldots, n$. The final shunt-only BPF circuit model with identical $L C$ components is shown in Fig. 4. The shunt inductor $(L)$ and the shunt capacitor $(C)$ of Fig. 4 were determined using (3), where $Z_{0}$ is the $50 \mathrm{Ohms}$ impedance due to the input and output terminations, and $\omega_{0}$ is the angular centre frequency of the BPF. The new J-inverter values of Fig. 4 were obtained using (4). The filter circuit model was simulated using the Agilent Advanced Design System (ADS) circuit simulator. Before performing the simulation, the couplings between resonators were modelled using the method presented in [12], which involves replacing each J-inverter on the circuit model with a pi-network of capacitors as shown in Fig. 5. The capacitance value, $C_{J}$, of each capacitor contained in the pi-network was determined using (5). The numerical design parameters for the BPF are given in Table II. The simulation results of the Chebyshev BPF circuit model are shown in Fig. 6. The results clearly show that the filter has its center frequency at $1.684 \mathrm{GHz}$ and its minimum return loss at $20 \mathrm{~dB}$ as designed.

$$
\begin{gathered}
J^{\prime}{ }_{01}=J_{n, n+1}^{\prime}=1, J_{m, m+1}^{\prime}=\sqrt{\frac{g_{1}^{2}}{g_{m} g_{m+1}}} \\
C=\frac{g_{1}}{\omega_{0} Z_{0} F B W}, L=\frac{Z_{0} F B W}{g_{1} \omega_{0}}
\end{gathered}
$$

$$
\begin{gathered}
J_{m, m+1}^{\prime}=\frac{J_{m, m+1}^{\prime}}{Z_{0}} \\
C_{J}=\frac{J}{\omega_{0}}
\end{gathered}
$$

TABLE II. 3-POLE CHEBYSHEV BPF DESIGN PARAMETERS

\begin{tabular}{|l|l|c|c|c|c|}
\hline Filter & $\mathbf{f}_{\mathbf{0}}[\mathbf{G H z}]$ & $\mathbf{L}[\mathbf{n H}]$ & $\mathbf{C}[\mathbf{p F}]$ & $\mathbf{J}_{\mathbf{0 1}}$ & $\mathbf{J}_{\mathbf{1 2}}$ \\
\hline BPF & 1.684 & 0.222 & 40.2424 & 0.02 & 0.0176 \\
\hline
\end{tabular}

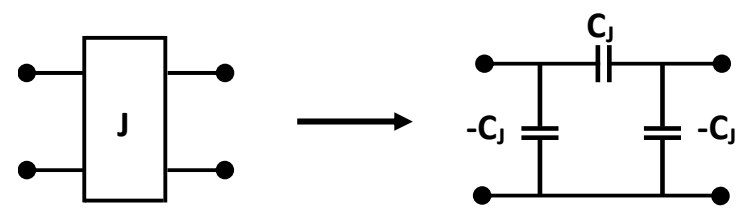

Fig. 5. J-inverter modelled as a pi-network of capacitors. 


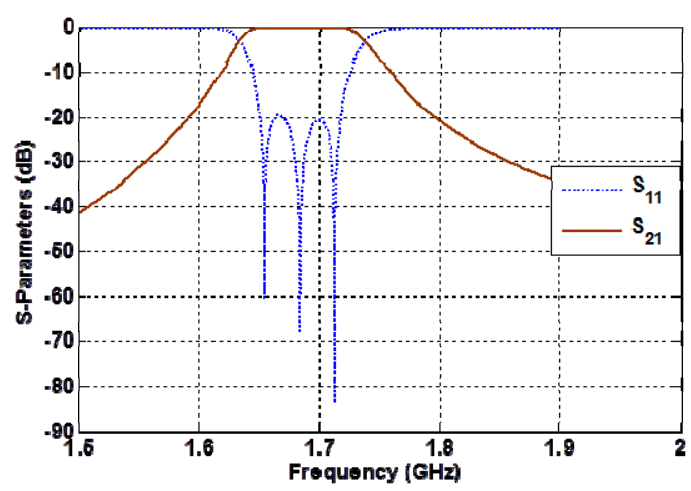

Fig. 6. Simulation results of the filter circuit model.

\section{SIW BANDPASS FILTER DESIGN}

The SIW cavity for the Chebyshev BPF was designed to resonate at the $\mathrm{TE}_{101}$ mode resonance frequency, $\mathrm{f}_{0}$ of 1.684 $\mathrm{GHz}$ using (1). The design was made on the Rogers RT/Duroid $6010 \mathrm{LM}$ substrate with $\varepsilon_{\mathrm{r}}=10.8, \mathrm{~h}=1.27 \mathrm{~mm}$ and $\mu_{\mathrm{r}}=1$. Other design parameters were chosen as follows: $d=2 \mathrm{~mm}, \mathrm{p}$ $=3.725 \mathrm{~mm}, \mathrm{w}=37.25 \mathrm{~mm}$, and $l=37.25 \mathrm{~mm}$. All electromagnetic (EM) simulations were carried out using the finite-element method (FEM) of the Keysight electromagnetic professional (EMPro) 3D simulator. A loss tangent, $\tan \delta=$ 0.0023 was assumed for the substrate, and a conductivity, $\sigma=$ $5.8 \times 10^{7} \mathrm{~S} / \mathrm{m}$ for all the metals (copper) with 17 microns $(\mu \mathrm{m})$ thickness. According to [15], commercial PCB-based SIW filters depends on large amount of metallic vias that require high density meshing and long simulation time for the fullwave simulations to converge. In order to achieve faster simulation results convergence, each metallized via was implemented as an octadecagon (18 sided polygons) instead of circles.

The coupling coefficient, $\mathrm{k}$, between each pair of SIW cavities were determined using the technique shown in Fig. 7 and using (6), where $f_{1}$ and $f_{2}$ are the eigenmodes from simulating a pair of SIW cavities. The value of $\mathrm{k}$ that was used to achieve the test filter is $\mathrm{k}=0.041$ at $\mathrm{s}=13.05 \mathrm{~mm}$. The external quality factor, $\mathrm{Q}_{\mathrm{ext}}$ of the filter was based on the method shown in Fig. 8. The length, $y_{3}$ corresponding to the 50 Ohms impedance of the microstrip transmission line was determined using the widely known formulations reported in [12]. The impedance at the SIW end of the transition was achieved by adjusting the lengths, $x_{1}$ and $y_{2}$, until the required filter $Q_{\text {ext }}$ value of 21.21 was achieved. The complete FEM layout of the SIW BPF that was simulated using the Keysight EMPro 3D simulator is shown in Fig. 8, with all the physical dimensions for the filter indicated. The simulation results of the proposed filter are presented in Fig. 9. It could be seen from the simulation results that the center frequency of the filter is about $1.68 \mathrm{GHz}$ as designed. The simulated insertion loss $\left(\mathrm{S}_{21}\right)$ of 1.3 $\mathrm{dB}$ and the simulated return loss $\left(\mathrm{S}_{11}\right)$ of $15 \mathrm{~dB}$ were achieved.

$$
k=\frac{f_{2}^{2}-f_{1}^{2}}{f_{2}^{2}+f_{1}^{2}}
$$

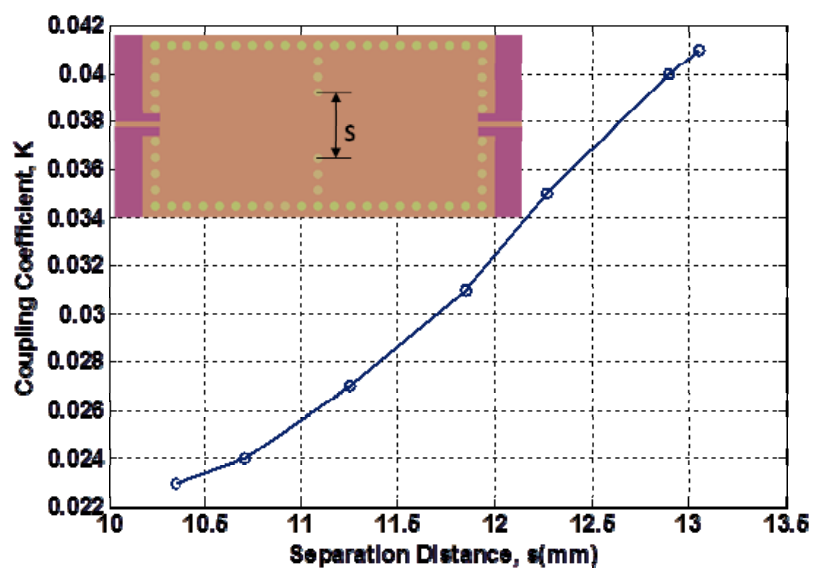

Fig. 7. Technique for achieving the coupling coefficient of a pair of substrate integrated waveguide cavities.

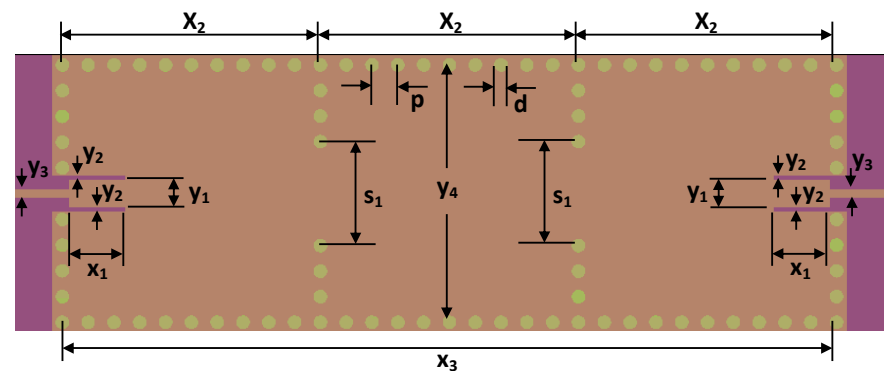

Fig. 8. Substrate integrated waveguide bandpass filter layout with physical dimensions in $\mathrm{mm}\left(\mathrm{d}=2.0, \mathrm{p}=3.725, \mathrm{~s}_{1}=13.05, \mathrm{x}_{1}=8.125, \mathrm{x}_{2}=37.25, \mathrm{x}_{3}=\right.$ $\left.111.75, \mathrm{y}_{1}=3.9, \mathrm{y}_{2}=0.7, \mathrm{y}_{3}=1.1, \mathrm{y}_{4}=37.25\right)$.

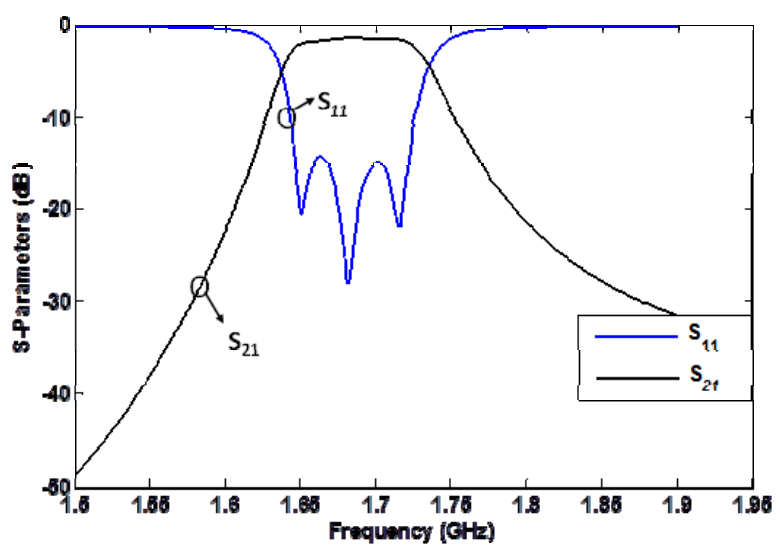

Fig. 9. Simulation responses of the proposed substrate integrated waveguide bandpass filter.

\section{FABRICATION AND MEASUREMENT}

The low cost SIW bandpass filter was fabricated using the same material employed in the FEM simulation. The fabrication was based on the low cost printed circuit board (PCB) milling process. The photograph of the fabricated filter is shown in Fig. 10. To allow for the measurement of the filter, 
two SMA (Sub-Miniature version A) connectors were fitted onto the input and output ports as shown. The testing and measurement were carried out using the Agilent Vector Network Analyzer. Fig. 11 shows the measurement results indicating that an insertion loss $\left(\mathrm{S}_{21}\right)$ of $1.3 \mathrm{~dB}$ and a return loss $\left(\mathrm{S}_{11}\right)$ of $16 \mathrm{~dB}$ were achieved. The measurement and simulation results are jointly presented in Fig. 12 for ease of comparison. It is clear from Fig. 12 that the simulation and measurement results are in good agreement.

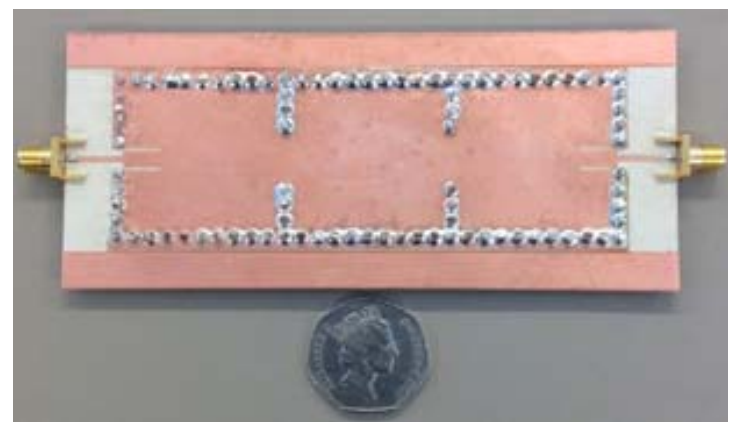

Fig. 10. Photograph of the fabricated bandpass filter

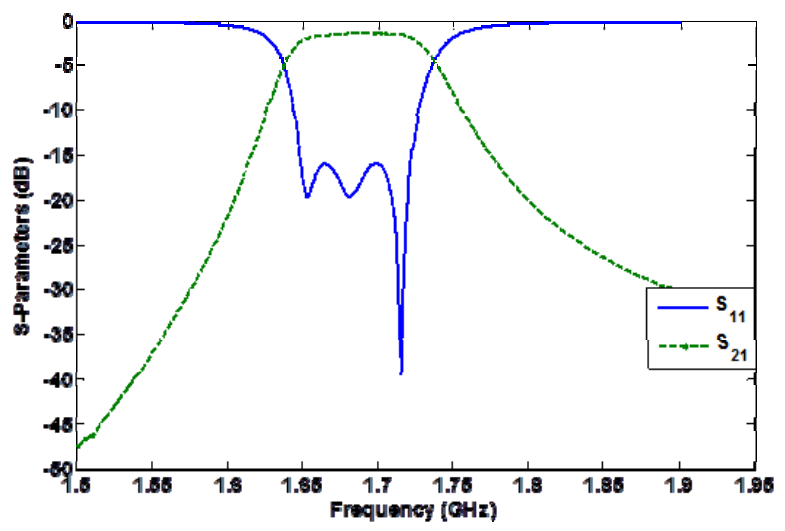

Fig. 11. Measurement results of the proposed bandpass filter.

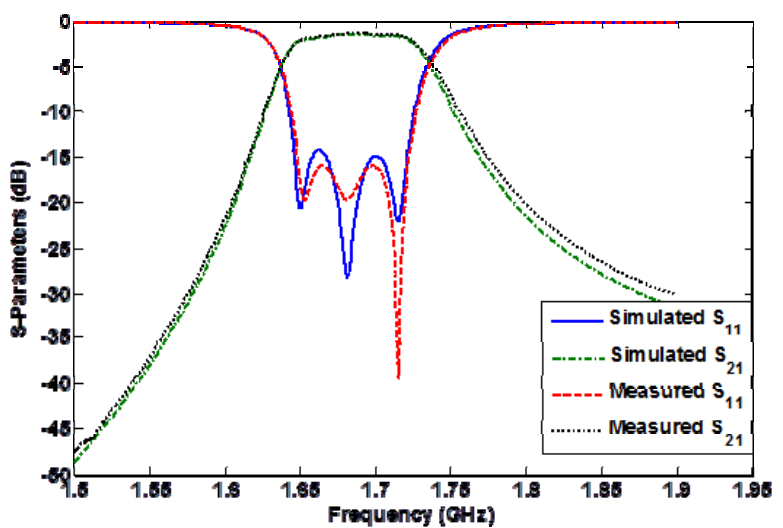

Fig. 12. Comparison of simulation and measurement results of the proposed substrate integrated waveguide bandpass filter.

\section{CONCLUSION}

A SIW Chebyshev bandpass filter using the low cost, commercially available PCB technology has been presented. The coplanar-to-SIW connection employed in the design is new as it involves two different stages of impedances. The low impedance at the SIW end of the transition makes for easy realization of the filter $Q_{\text {ext. }}$ The design has been experimentally validated and results presented. The simulation and measurement results show that an insertion loss of $1.3 \mathrm{~dB}$ was achieved across the band. The simulated and measured return losses of $15 \mathrm{~dB}$ and $16 \mathrm{~dB}$ respectively were achieved. The simulated and measured results show good agreement with the passband centered at $1.684 \mathrm{GHz}$ as expected.

\section{REFERENCES}

[1] D. Deslandes, and $\mathrm{K}$. Wu, "Integrated microstrip and rectangular waveguide in planar form," IEEE Microw. Wireless Compon. Lett., vol. 11 , no. 2, pp. 68-70, Feb. 2001.

[2] H. J. Tang, W. Hong, J.-X. Chen, G. Q. Luo, and K. Wu, "Development of millimeter-wave planar diplexers based on complementary characters of dual-mode substrate integrated waveguide filters with circular and elliptic cavities', IEEE Trans. Microw. Theory Techn., vol. 55, no. 4, pp. 776-782, Apr. 2007.

[3] H. Chu, Y. X. Guo, Y. L. Song, and Z. L. Wang, “40/50 GHz diplexer design in LTCC technology," IET Electron. Lett., vol. 47, no. 4, pp. 260262. Feb. 2011.

[4] C.-W. Tang, and S.-F. You, "Design methodologies of LTCC bandpass filters, diplexer, and triplexer with transmission zeros," IEEE Trans. Microw. Theory Techn., vol. 54, no. 2, pp. 717-723, Feb. 2006.

[5] D. Orlenko, K. Markov, S. Royak, A. Gordiyenko, O. Chernyakov, T. Kerssenbrock, G. Sevskiy, and P. Heide, "Novel high-rejection LTCC diplexers for dual-band WLAN applications," in IEEE MTT-S Int. Microw. Symp. Dig., Jun. 2005, pp. 727-730.

[6] K. Kunihiro, T. Yamanouchi, Y. Miyazaki, Y. Aoki, K. Ikuina, T. Ohtsuka, and H. Hida, "A diplexer-matching dual-band power amplifier LTCC module for IEEE $802.11 \mathrm{a} / \mathrm{b} / \mathrm{g}$ wireless LANs," in IEEE Radio Freq. Integr. Circuits Symp. Dig., Jun. 2004, pp. 303-306.

[7] S.-W. Wong, K. Wang, Z.-N. Chen, and Q.-X. Chu, "Electric coupling structure of substrate integrated waveguide (SIW) for the application of 140-GHz bandpass filter on LTCC," IEEE Trans. Comp. Pack. Manuf. Technol., vol. 4, no. 2, pp. 316-322, Feb. 2014.

[8] S. Dalmia, G. White, V. Sundaram, and M. Swaminathan, "Design of quasi-lumped element diplexer for multiple wireless applications using liquid crystalline polymer (LCP) based substrates," in Proc. Eur. Microw. Conf., Oct. 2004, pp. 737-740.

[9] A. O. Nwajana, and K. S. K. Yeo, "Microwave diplexer purely based on direct synchronous and asynchronous coupling," Radioengineering, vol. 25, no. 2, pp. 247-252, Jun. 2016.

[10] X.-P. Chen, and K. Wu, "Substrate integrated waveguide filter: basic design rules and fundamental structure features," IEEE Microw. Mag., vol. 15 , no. 5, pp. 108-116, Jul.-Aug. 2014.

[11] D. Deslandes, and $\mathrm{K}$. Wu, "Single-substrate integration technique of planar circuits and waveguide filters," IEEE Trans. Microw. Theory Techn., vol. 51, no. 2, p. 593-596, Feb. 2003.

[12] J.-S. Hong, Microstrip Filters for RF/Microwave Applications, New York, NY, USA: Wiley, 2011.

[13] S. H. Han, X. L. Wang, Y. Fan, Z. Q. Yang, and Z. N. He, "The generalized Chebyshev substrate integrated waveguide diplexer," Progress Electromagn. Res., vol. 73, pp. 29-38, 2007.

[14] K. S. K. Yeo and A. O. Nwajana, "A novel microstrip dual-band bandpass filter using dual-mode square patch resonators," Progress Electromagn. Res. C, vol. 36, pp. 233-247, Jan. 2013.

[15] Z.-C. Hao, W.-Q. Ding, and W. Hong, "Developing low-cost W-band SIW bandpass filter using the commercially available printed-circuitboard technology," IEEE Trans. Microw. Theory Techn., vol. 64, no. 6, pp. 1775-1786, Jun. 2016. 